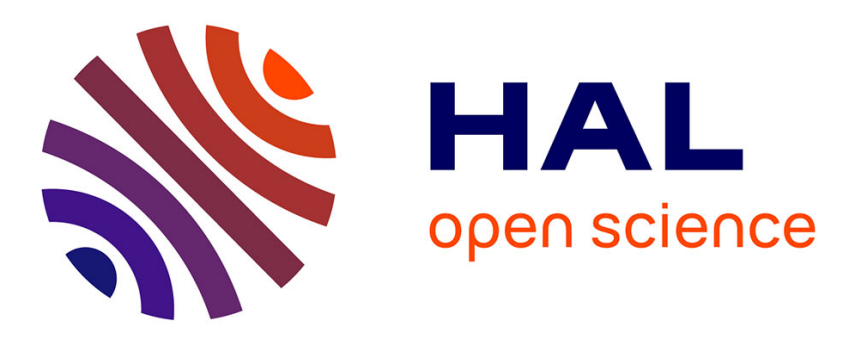

\title{
Les signalements profanes de clusters de cancers: épidémiologie populaire et expertise en santé environnementale
}

\author{
Marcel Calvez
}

\section{- To cite this version:}

Marcel Calvez. Les signalements profanes de clusters de cancers: épidémiologie populaire et expertise en santé environnementale. Sciences Sociales et Santé, 2009, 27 (2), pp.79-106. 10.1684/sss.2009.0206 . halshs-00402873

\section{HAL Id: halshs-00402873 \\ https://shs.hal.science/halshs-00402873}

Submitted on 6 Oct 2010

HAL is a multi-disciplinary open access archive for the deposit and dissemination of scientific research documents, whether they are published or not. The documents may come from teaching and research institutions in France or abroad, or from public or private research centers.
L'archive ouverte pluridisciplinaire HAL, est destinée au dépôt et à la diffusion de documents scientifiques de niveau recherche, publiés ou non, émanant des établissements d'enseignement et de recherche français ou étrangers, des laboratoires publics ou privés. 


\title{
Les signalements profanes de clusters de cancers : «Epidémiologie populaire» et expertise en santé environnementale ${ }^{1}$
}

\author{
Marcel Calvez ${ }^{*}$
}

\begin{abstract}
Résumé : Les signalements profanes de clusters de cancers combinent la perception d'une fréquence inhabituelle de pathologies de type cancéreux par une population, l'incrimination d'installations industrielles dans ces pathologies et le signalement auprès des autorités de santé pour entreprendre des études scientifiques et des actions de contrôle des risques de santé. Le plus souvent, la perception d'un agrégat n'est pas validée par les études épidémiologiques menées sur le site. La population est alors conduite à développer sa connaissance du problème de santé au travers d'une démarche que, à la suite de Phil Brown, on qualifie d'épidémiologie populaire. L'article s'appuie sur l'ethnographie de trois signalements de clusters (Saint-CyrL'École, Vincennes, Nivillac) pour analyser cette épidémiologie populaire, sa revendication à l'expertise et son ancrage social et culturel.
\end{abstract}

Mos-clés : Epidémiologie populaire, clusters profanes, cancers, santé
environnementale, expertise

L'épidémiologie utilise le terme anglais «cluster» ou l'expression «agrégat spatiotemporel » pour désigner "le regroupement dans le temps et l'espace de cas de maladies, de symptômes ou d'événements de santé au sein d'une population localisée » (InVS, 2005 :5). Parmi les différents agrégats de pathologies, les clusters de pathologies non infectieuses retiennent l'attention parce qu'ils articulent le plus souvent une sensibilité affirmée à l'égard des cancers et des préoccupations relatives à des installations industrielles ou à des sites pollués. Ce croisement de préoccupations se manifeste dans l'importance que ces clusters revêtent parmi les différents signalements d'agrégats. Aux USA, le NCEH a reçu 405 signalements de clusters de cancers entre mai 2002 et janvier $2004^{2}$. En France, 1'InVS

\footnotetext{
${ }^{1}$ Les recherches sur les signalements profanes de clusters ont été financées par l'ADEME (Agence pour le développement et la maîtrise de l'énergie) \# 0410 C 0041, le Programme CNRS SBSS (Sciences biomédicales Santé Société) \# 08/2005/DR03, et le Ministère de la recherche (ATC Environnement-Santé) \# 04 5667. Elles ont associé Denis Bard et Patricia Loncle (EHESP, Rennes) Le terrain a été réalisé par Marcel Calvez et Sarah Leduc.

Une première version de cet article a été présentée au colloque «L'expertise comme objet flou ? », CRAPE -IEP de Rennes, mars 2008.

${ }^{2}$ Source NCEH - CDC. Le NCEH (National Center for Environmental Health ) a été établi aux CDC (Centers for Disease Control and Prevention) en mai 2002 pour centraliser et suivre les préoccupations de santé environnementale.
} 
(Institut de veille sanitaire) a recensé 38 signalements de clusters entre 1997 et 2002 (Tillaut, 2005). Dans trois cas sur quatre, ces signalements concernent des cancers. Dans deux cas sur trois, une source environnementale est mise en cause, le plus souvent un site polluant. Dans un peu moins de la moitié des cas, une association locale est impliquée dans le signalement, soit qu'elle en ait été à l'origine ou qu'elle s'en soit saisie. Des investigations sanitaires ont été réalisées sur la plupart de signalements par l'InVS ou les CIRE (Cellules interrégionales d'épidémiologie) en prenant appui sur les critères mis en évidence dans la littérature internationale ${ }^{3}$. Selon ces critères, aucun des signalements ne peut être qualifié de cluster : les excès perçus de cas restent le plus souvent dans les limites des cas statistiquement attendus ; aucune hypothèse relative à une origine environnementale des pathologies ne peut être émise ou validée.

$\mathrm{Si}$, du point de vue des autorités de santé publique, la question soulevée par le signalement d'un cluster est close par une expertise épidémiologique non concluante, il n'en est pas de même du point de vue des acteurs qui le signalent ${ }^{4}$. Le signalement auprès des autorités sanitaires procède en effet de préoccupations qui conduisent ces acteurs à produire une connaissance sur des risques de santé environnementale et à interpeller les autorités de santé. C'est cette production d'une connaissance sur les maladies et leurs causes que, à la suite de $\mathrm{Ph}$. Brown, on qualifie d'épidémiologie populaire.

\section{L'épidémiologie populaire, l'expert et le profane}

Brown a mené des recherches pionnières sur les signalements de clusters aux USA, en premier lieu sur les cas de leucémie à Woburn dans le Massachusetts. (Brown, Mikkelsen, 1990 ; Brown, 1992, 1994). Dans l'analyse de cette mobilisation, il utilise la notion d'épidémiologie populaire pour désigner «le processus par lequel des profanes rassemblent des données [scientifiques] et d'autres informations, et orientent et mobilisent la connaissance et les ressources des experts pour comprendre l'épidémiologie de la maladie. Dans certaines de ses actions, l'épidémiologie populaire est analogue à l'épidémiologie scientifique, comme lorsque des profanes mènent des enquêtes de santé. Toutefois, l'épidémiologie populaire est plus qu'une participation publique à l'épidémiologie traditionnelle, dans la mesure où elle met en avant les facteurs sociaux et structurels comme une composante de la chaîne causale de la maladie. De plus, elle implique des mouvements sociaux, utilise des approches politiques et judiciaires et remet en cause les postulats de base de l'épidémiologie traditionnelle, de l'évaluation des risques et des réglementations de santé publique » (Brown, 1992 : 269).

L'épidémiologie populaire trouve une première expression dans les signalements profanes de clusters. Lorsque ces signalements donnent lieu à une expertise officielle, ils peuvent être disqualifiés. Les personnes qui ont signalé le cluster se trouvent placées face à un dilemme : soit accepter l'expertise et renoncer à leur propre représentation du problème, soit contester l'expertise et donner un retentissement public au problème de santé. Dans ce cas, la

\footnotetext{
${ }^{3}$ Pour qu'il y ait un cluster, il faut qu'il y ait une pathologie unique et clairement définie ; que la maladie en cause présente une forme différente des cas précédemment recensés ; que la population affectée soit différente de la population habituellement atteinte par cette maladie ; qu'un facteur de risque connu soit présent ; que le risque relatif soit élevé ; et qu'une exposition spécifique à un facteur de risque soit identifiée (InVS, 2005).

${ }^{4}$ Ces acteurs du signalement renvoient à l'un des types de "lanceurs d'alertes" qu'analysent Chateauraynaud et Torny à Gammaville et à Penly (1999 : 264-269). La recherche s'intéresse de façon prioritaire à la construction d'une connaissance et à son ancrage social, ce qui a conduit à rester en retrait par rapport à la question de l'alerte que ces auteurs abordent de façon systématique et à ne pas reprendre la désignation qu'ils utilisent.
} 
réalité du cluster devient l'objet d'un conflit de perspectives qui oppose deux façons de produire de la connaissance et d'élaborer des interprétations des problèmes de santé. Du point de vue des experts mandatés, ce conflit se manifeste par la contestation d'un savoir scientifique éprouvé et reconnu au nom de sentiments ou d'émotions considérés comme légitimes, mais extérieurs aux procédures de la connaissance que les profanes ne maîtrisent pas ou auxquelles ils ne sont pas acculturés ${ }^{5}$. D'où la nécessité d'une communication pour faire comprendre au public ce qu'est l'activité d'expertise. Du point de vue des acteurs locaux qui signalent le cluster, le conflit procède de la mise en cause de la réalité du problème de santé par les experts ${ }^{6}$. Ce conflit de perspectives renvoie à deux univers sociaux largement divergents. Pour ceux qui signalent le cluster, sa réalité se renforce à la critique des experts, de leurs méthodes et de leurs résultats. Malgré leurs efforts de communication et de vulgarisation des résultats, les experts considèrent ne pas être entendus. A la demande de certitudes, ils apportent des réponses que les plaignants jugent incertaines ou ambiguës. Dans de nombreux cas, le conflit de perspectives reste à l'état latent ; les différents protagonistes ne se rencontrent pas et ne sont pas conduits à expliciter ce qui fait problème dans un contexte où des personnes sont malades ou décèdent. Dans quelques cas, ce conflit acquiert une expression publique lors de situations au cours desquelles les experts et les plaignants sont conduits à dire et explorer les causes de leurs désaccords quant aux causes de ces pathologies. C'est alors que l'épidémiologie populaire acquiert une expression publique qui la rend accessible à l'analyse sociologique. Elle objective des questions ou des problèmes dans un langage de santé publique qui est plus ou moins contesté par les professionnels de ce champ.

De façon conventionnelle, les sociologues abordent de tels conflits de perspective au travers de la dichotomie entre l'expert et le profane. Cette dichotomie trouve son origine dans l'analyse des professions développée par E. C. Hughes et ses élèves au cours des années 1950 (Hughes, 1952 ; 1996). Elle reprend la distinction religieuse entre le sacré et le profane pour souligner que chaque activité, quelle que soit la nature des tâches réalisées, se délimite un domaine de prestige et de compétences qui est conforme aux normes sociales en vigueur et cherche à exercer un contrôle exclusif de ce domaine. Cette approche de la dimension morale du travail vaut autant pour le janitor, qui s'occupe de la maintenance des immeubles, que pour le médecin. En montrant que chaque profession et chaque métier se définissent par la délimitation stratégique d'un domaine exclusif en dehors du monde profane, Hughes entend rompre avec l'approche développée par Parsons (1951) qui voit dans les professions un aboutissement organisationnel de la diffusion du progrès scientifique et dans la rationalité un attribut associé à ce progrès. Il introduit ainsi l'idée d'une divergence de points de vue entre le profane et l'expert qui est fondée dans une différence d'expériences ou de pratiques sociales et non dans la rationalité du professionnel et l'irrationalité du profane.

Brown critique l'exclusion des profanes des décisions en matière de santé publique (Brown, Mikkelsen : 1990, 146 et sq). Il voit cette exclusion comme une stratégie de contrôle de la production de connaissances par des professionnels qui défendent leurs institutions et leurs statuts. Selon lui, les enjeux professionnels en cause dans cette exclusion sont traduits en termes de valeur de la connaissance scientifique pour tenir les profanes à distance. "Les revendications des professionnels à l'autonomie dans la direction d'enquêtes scientifiques reposent en partie sur l'idée que l'épidémiologie scientifique peut atteindre un degré élevé de

\footnotetext{
${ }^{5}$ Voir par exemple Robinson (2002) qui exprime la position du Conseil américain sur la science et la santé (American council on science and health), également InVS (2005).

${ }^{6}$ Des films comme Civil action (Préjudice en VF) (1999) ou Erin Brockovitch (2000) ont largement popularisé ces oppositions en mettant en en scène le besoin de justice pour les victimes contre le pouvoir des pollueurs et leur ont donné une expression culturelle qui imprègne largement les façons courantes d'aborder les questions posées par les clusters.
} 
certitude. En fait, la connaissance scientifique est pleine d'incertitudes, en particulier dans le cas de la contamination par des déchets toxiques »(Ibid.: 147). Il considère donc que la connaissance populaire est légitime dans le contexte politique de crises comme celles relatives aux déchets, dans lesquelles les implications en matière de santé ne sont pas connues avec certitude. C'est dans cette perspective qu'il souligne la similitude de démarche basée sur des observations de terrain entre la recherche des causes des leucémies par les résidents de Woburn et la découverte des origines du choléra de Londres en 1854 par John Snow, considéré comme l'un des initiateurs de la démarche scientifique en épidémiologie (Coura, 2004). Cette connaissance est pertinente car «les gens ont souvent accès à des données à propos d'eux-mêmes et de leur environnement qui ne sont pas accessibles aux scientifiques » (Brown, Mikkelsen : 1990 : 127), ce dont témoignent les signalements de clusters de cancers ou de risques toxiques. Ils sont également en capacité de faire des observations et des recoupements entre situations pour identifier des facteurs d'exposition qui s'avèrent, à l'expérience, moins biaisés que ce que les autorités affirment d'emblée. Ces arguments conduisent Brown à appliquer la notion d'épidémiologie populaire aux pratiques des acteurs locaux qui s'impliquent dans la découverte et la recherche de causes de maladies.

L'approche développée par Brown insiste sur la collecte de données et en souligne la pertinence, ce qui lui permet de mettre l'épidémiologie populaire sur un plan comparable à l'épidémiologie scientifique. Dans la même lignée, S. Kroll-Smith et H.H. Floyd parlent d'un «mouvement dans lequel des gens ordinaires s'approprient les techniques et les concepts d'un système expert, les relient à leur expérience personnelle et revendiquent une reconnaissance institutionnelle de leurs maux dans un langage de rationalité instrumentale » (Kroll-Smith, F1oyd, 1997 : 192). Ces approches de l'épidémiologie populaire considèrent l'appropriation populaire des techniques scientifiques ; mais elles ne s'interrogent pas sur les connaissances tacites qui les sous-tendent, c'est-à-dire les façons dont les acteurs les utilisent et dont ils élaborent des explications (Collins, Evans, 2002; 2007). Elles laissent ainsi largement de côté les divergences dans les styles de travail épidémiologique tout comme les modalités d'établissement des relations de causalité qui, plus que la collecte de données de terrain, constitue la pierre d'achoppement entre les différentes pratiques de l'épidémiologie. Dans les études réalisées aux Etats-Unis d'Amérique, cette moindre attention peut être imputable au fait que les signalements étudiés sont présentés devant la justice qui statue sur leur validité. "Les définitions légales de la causalité [...] sont d'abord déterminées par l'interprétation judiciaire des témoignages scientifiques »(Brown, 1995 : 109) ${ }^{7}$. On peut toutefois remarquer que, avant que le juge valide ou non leurs demandes, les profanes élaborent une relation de causalité entre un problème de santé et un polluant, qu'ils jugent suffisamment probante pour engager des actions publiques. La construction de cette relation de causalité doit être étudiée pour aborder de façon satisfaisante l'épidémiologie populaire.

L'établissement de relations de causalité peut être abordée d'un point de vue sociologique à partir de leur ancrage dans des collectifs de pensée au sein desquels une façon de voir est développée et devient légitime (Kuhn, 1983 ; Douglas, 1999 ; Fleck, 2005). Si les collectifs profanes et scientifiques de l'épidémiologie sont des communautés de pratiques dans lesquelles les individus peuvent être socialisés, ils présentent des différences qui ne tiennent pas simplement au statut social qui leur est alloué. Les collectifs profanes rassemblent des personnes aux trajectoires différentes qui se trouvent ensemble parce qu'elles partagent un même territoire et le plus souvent un style de vie comparable, et qui se mobilisent parce qu'elles partagent une représentation commune d'un risque de santé. Les

\footnotetext{
${ }^{7}$ Dans le contexte français, les signalements de clusters qui ont donné lieu à des actions en justice sont rares. A notre connaissance, seuls les signalements de Gilly-sur-Isère et de Nivillac, tous deux relatifs à des incinérateurs, ont donné lieu à des procédures devant les tribunaux.
} 
collectifs scientifiques rassemblent des personnes qui ont été "socialisées dans les aspects formels d'une discipline scientifique tout en acquérant, dans le même temps, la connaissance sociale et culturelle tacite qui est requise pour appliquer et utiliser ces faits dans de nouveaux contextes »(Evans 2008 : 283). L'expertise profane a son ancrage dans la singularité d'un territoire de vie et dans une condition sociale partagée ; c'est par l'intermédiaire de cette condition partagée que les représentations que le collectif développe deviennent des connaissances plausibles, puis valides en tant qu'elles expriment une expérience commune. L'expertise scientifique prend appui sur une communauté professionnelle et sur des règles générales qui permettent d'agir de façon disciplinairement appropriée dans des contextes différenciés et de valider les connaissances élaborées. Ce sont ainsi les expériences sociales dans lesquelles les deux pratiques de l'épidémiologie s'ancrent et les connaissances et critères de jugements tacites qu'elles véhiculent qui divergent.

L'épidémiologie populaire peut ainsi être regardée comme une modalité de production de connaissances sur des questions de santé qui procède de collectifs de pensée, constitués à partir d'agrégats de pathologies perçues comme inhabituelles ou atypiques, et qui sont en dehors de l'activité institutionnelle d'expertise. Elle se présente, non pas comme un corpus unifié de règles d'établissement des faits, mais comme un ensemble relativement hétérogène de savoirs et de procédures qui résulte de la diversité des expériences sociales et des conditions concrètes dans lesquelles les connaissances des questions de santé se sont formées.

Pour présenter cette épidémiologie populaire, on situera tout d'abord les trois signalements d'agrégats de cancers qui ont été l'objet d'une étude de terrain. Puis, on s'intéressera aux conditions de formation des signalements. On discutera ensuite la démarche par laquelle une relation de causalité est élaborée entre les installations industrielles mises en cause et les agrégats de cancers. On s'intéressera enfin à la revendication d'expertise en montrant qu'elle oscille entre deux stratégies différentes d'ancrage de la connaissance profane. En conclusion, on s'interrogera sur les réalités sociales et culturelles que cette épidémiologie populaire des clusters cherche à exprimer.

\section{Les trois signalements de clusters étudiés}

L'étude a porté sur trois sites français dans lesquels il y a eu des signalements d'agrégats de cancers associés à l'incrimination d'installations industrielles entre 2000 et $2002^{8}$. Le choix de ces trois sites fait suite une première enquête qui est partie des signalements recensés par l'InVS entre 1997 et 2002 (Tillaut, 2005) et qui a considéré ceux pour lesquels les données étaient accessibles et pour lesquels l'agrégat de cancers ou la cause incriminée était l'objet de désaccords. Les signalements d'agrégats en milieu de travail ou en milieu hospitalier n'ont pas été considérés : en ayant cours dans un système de travail organisé, ils ouvrent en effet sur des questions relatives aux risques et à leur attribution différentes de celles qui concernent la vie courante. Le choix s'est porté sur trois sites différents afin de prendre en compte une hétérogénéité des sources incriminées et une diversité des populations touchées. Les possibilités pratiques de réaliser une enquête ont été prises en compte ; une attention particulière a été prêtée à une possible saturation des terrains par les chercheurs, ce qui a conduit à exclure le cas de Gilly-sur-Isère en Savoie, très largement médiatisé après 2001, mais aussi objet d'enquêtes épidémiologiques et sociologiques commandités par l'InVS (Salomon, 2003 ; Thabuis, Schmitt, 2006).

\footnotetext{
${ }^{8}$ Ces trois cas sont présentés dans l'ouvrage de A. Cicollela et D. Benoit Browaeys (2005 : respectivement p. 147-148, 176-179 et 169-170) qui reprend le point de vue d'un acteur local très impliqué dans le signalement.
} 
Une enquête de type ethnographique a été menée auprès des acteurs locaux qui ont été directement impliqués dans le signalement. Dans chacun de sites, elle a concerné entre dix et quinze personnes ; celles qui étaient au coeur du signalement ont été rencontrées à plusieurs reprises; les archives qu'elles ont pu mettre à notre disposition ont été étudiées. Cette enquête a été complétée par une recherche documentaire sur la publicité donnée à ces agrégats dans les médias écrits et audiovisuels et sur internet. L'accès aux terrains a été initialement marqué par une méfiance de la part des acteurs locaux, dans un contexte où leur signalement était disqualifié par les experts qui concluaient à l'absence de cluster'. Le temps long d'enquête ethnographique et l'écoute des personnes dans ce qu'elles avaient à dire sur ces signalements ont été essentiels pour dépasser cette situation première. La posture de recherche a consisté à chercher à comprendre les processus par lesquels la réalité des clusters est construite localement et à rendre compte des expériences sociales dans lesquelles elle s'ancre. Les analyses des experts ont été regardées dans ce qu'elles impliquaient localement et replacées dans l'analyse du processus local de signalement. Les enquêtes de terrain ont débouché sur des monographies qui, après une chronologie du signalement, ont eu pour objet de caractériser les réseaux d'acteurs par lesquels il s'est formé, les modalités par lesquelles la mise en cause d'une installation s'est faite, les actions mises en œuvre en direction des autorités ou des experts.

A Saint-Cyr-l'École (Yvelines), le signalement a pour cadre un quartier pavillonnaire, occupé par des catégories populaires anciennement établies et le plus souvent en retraite, et par des classes moyennes récemment arrivées dans ce marché de report de l'Ouest parisien avec des enfants en bas âge. La localisation du quartier sur un plateau, entre autoroute, chemin de fer et forêt, en fait une sorte d'enclave dans la ville. Le signalement a pour objet des antennes de relais de téléphonie mobile installées sur le toit d'un groupe scolaire (primaire et maternelle) en 1992 et 1997. Ces antennes sont mises en cause dans l'apparition de pathologies rares chez des enfants du quartier dès 2000, dans un contexte où les antennes relais et les téléphones portables deviennent un objet de préoccupation publique ${ }^{10}$. Trois mères d'élèves arrivées dans le quartier depuis quelques années, sont à l'origine de cette mise en cause. Elles demandent l'interdiction de nouvelles antennes et commencent à mobiliser localement les parents d'élèves. Par l'intermédiaire des associations de parents, elles réalisent une enquête à la fin de 2001. En utilisant un questionnaire élaboré par un scientifique sur les pollutions électromagnétiques, elles cherchent à objectiver les effets des ondes sur la santé et interrogent la population vivant aux alentours des antennes. Elles recensent 11 cas de cancers chez des enfants de moins de 14 ans, ainsi que des troubles de santé divers (maux de têtes, saignements, etc.). En 2002, elles initient un collectif d'associations qui se mobilise contre le projet d'implantation de 6 nouvelles antennes dans le quartier. Le maire de la commune de

\footnotetext{
${ }^{9}$ Cette méfiance a été plus grande à Saint-Cyr-l'École car l'enquête que nous avons menée s'est déroulée après un travail réalisé par une équipe de sociologues qui avaient travaillé sur les mobilisations autour des antennesrelais (Borraz, Devigne, Salomon, 2004). Cette enquête a été menée en 2003, ce qui correspond au moment des tensions les plus fortes entre le collectif d'associations locales et les autorités sanitaires ; la nôtre a été menée en 2005, quelques mois après le rapport de l'InVS récusant le signalement local. L'étude de Borraz et al. a été financée en partie par les opérateurs de téléphonie mobile, ce qui a pu induire une suspicion locale, qui conduit les chercheurs à justifier dans un long préambule le caractère scientifique de leur démarche (p. 8 et sq). Borraz et al. soulignent que localement la préoccupation concernait la santé alors que leur objet d'étude concernait la téléphonie mobile. Comme avec les épidémiologistes, les acteurs locaux expriment un désaccord relatif à l'objet de recherche et aux façons de travailler de cette équipe qui, selon eux, ne s'est pas donné les moyens de les rencontrer. Il est probable que notre entrée par les questions de santé a facilité l'accès au terrain.

${ }^{10}$ En 1999, le rapport de P. Lannoye, vice-président de la commission de l'environnement, de la santé publique et de la protection des consommateurs au parlement Européen, sur les risques de la téléphonie mobile. En 2000, un colloque "Téléphones portables, danger pour la santé ?" est organisé par le groupe d'études Santé Environnement de l'Assemblée nationale.
} 
Saint-Cyr-l'École, nouvellement élu, prend le parti des plaignantes et interpelle la DGS (Direction générale de la santé) qui saisit l'InVS pour étudier la possibilité de réaliser une investigation concernant les risques sanitaires éventuels liés à l'exposition aux champs électromagnétiques de radiofréquence. L'InVS considère qu'une telle étude n'est pas légitime sur le plan scientifique mais suggère d'explorer le signal sanitaire et de voir si les pathologies déclarées et leurs modalités de survenue font suspecter un agrégat. Parallèlement, le collectif d'associations se mobilise pour demander la désactivation des antennes existantes. Les médias nationaux relayent leur action. Enfin, un opérateur saisit le ministère de la Santé pour demander que des réponses soient apportées à la municipalité et aux associations locales. En octobre 2002, une mission d'étude est confiée à la Ddass et l'InVs sur les pathologies qui pourraient résulter d'une exposition passive aux rayonnements des antennes. La CIRE ne considère pas les pathologies signalées comme constituant un regroupement de cas pouvant être expliqué par une exposition environnementale commune (Drass Ile-de-France et al., 2004 ; Dejour-Salamanca et al., 2005). L'InVS recommande de ne pas continuer les investigations. La présentation publique des résultats en 2005 conduit le collectif à remettre en cause les méthodes et les résultats de l'enquête, et à demander une nouvelle expertise. Entre temps, en avril 2003, les opérateurs démantèlent les antennes relais placées sur le groupe scolaire.

A Vincennes (Val de Marne), la suspicion d'un agrégat de cancers d'enfants a pour cadre l'école maternelle Franklin Roosevelt qui se situe à l'emplacement d'un ancien site industriel Kodak. Ce site a été reconverti en 1990 en une résidence privée de standing, composée de bureaux et d'immeubles d'habitations principalement en location. La résidence est majoritairement habitée par des familles de classes moyennes à revenus élevés, attirées par le confort de l'habitat, les services d'accueil offerts pour les enfants et la proximité immédiate de Paris et du Bois de Vincennes. Entre 1995 et 1999, trois cas de cancers sont diagnostiqués chez des enfants fréquentant l'école maternelle. La directrice de l'école alerte l'Inspection d'académie, puis la Mairie qui commande une étude, laquelle ne relève pas d'anomalie. Parallèlement, un directeur de recherche en toxicologie, militant contre l'amiante apprend l'existence de cas de cancers sur le site et sollicite l'intervention de l'InVS. L'InVS rend ses conclusions en mai 2000 ; elles établissent la possibilité d'un excès de cas sans confirmation statistique et n'identifient pas de risques environnementaux spécifiques. En prenant appui sur l'existence d'un quatrième cas de cancer et le décès d'un autre enfant non recensé dans le premier signalement, les parents d'élèves demandent des investigations complémentaires que l'InVS ne juge pas nécessaires. Avec des riverains du quartier sud de Vincennes, ils fondent un collectif «Vigilance Franklin» auquel est associé le toxicologue. Le collectif se donne pour but de «connaître l'ampleur de la pollution générée par l'ex-usine Kodak et de rechercher des liens possibles avec l'excès de cancers d'enfants recensé depuis 1995 ». Il obtient la mise en place d'un comité scientifique chargé d'étudier les demandes qu'il formule, d'établir un programme d'études épidémiologiques et environnementales, et d'auditionner des experts. Le toxicologue qui lui est associé démissionne très vite du comité, dénonçant des confusions d'intérêt et un refus de prendre en compte les demandes du collectif. Parallèlement, un comité de suivi rassemblant organismes et associations est mis en place. Une campagne de communication et d'information est entreprise par la Mairie. Pour éviter les pressions psychologiques et médiatiques sur les enfants, le maire décide de fermer l'école en juin 2001. L'étude épidémiologique, publiée en mai 2002, confirme un excès de cas sur la période 1995-1999, mais n'envisage pas l'exposition à un facteur environnemental susceptible d'expliquer cet excès dans une zone délimité et sur une période limitée (InVS, 2002). Un aléa statistique est alors invoqué. Par ailleurs, l'étude détaillée des risques conclut en juin 2003 à des risques très inférieurs aux risques acceptables. L'école est rouverte en 
janvier 2004. Des campagnes de surveillance environnementale sont entreprises entre 2004 et 2007.

A Nivillac (Sud-est du Morbihan), la source incriminée dans le signalement d'un excès de cancers est un incinérateur d'ordures ménagères de petite capacité géré par un SIVOM (Syndicat intercommunal à vocation multiple). Cet incinérateur est situé dans une zone agricole vieillissante à l'habitat dispersé, à 5 kms à l'Est d'une agglomération de Nivillac en pleine expansion. L'incinérateur a fonctionné de façon intermittente entre 1990 et août 1999 où il a été arrêté suite à un incendie. Son autorisation de fonctionnement a été suspendue par arrêté préfectoral, en mars 2000 à cause d'une absence de mise en conformité de l'installation. Au début 2001, un ambulancier, atteint d'un lymphome, fait part à son médecin traitant de l'impression d'une augmentation des cancers dans la région et met en cause l'incinérateur. Avec ses deux confrères de la maison médicale, récemment installés dans la région, ce médecin saisit la Ddass du Morbihan en mai 2001. Ils signalent avec prudence l'impression d'une augmentation des cancers et suggèrent une éventuelle relation avec l'incinérateur. La Ddass, considérant qu'il s'agit d'une population limitée exposée à un risque a priori faible, ne donne pas suite. L'ambulancier et un élu local, tous deux opposés au président du Sivom, collectent des informations sur les dysfonctionnements de l'incinérateur et établissent une carte de 200 cancers sur 10 ans qui, selon eux, lui sont imputables. La publicité donnée à l'incinérateur de Gilly-sur-Isère à la fin de 2001 propulse le cas de Nivillac sur la place publique, sans que cela soit accompagné d'une mobilisation locale. A la demande du Ministère de la santé, la Ddass est appelée à mener des études qui concluent en juillet 2002 à l'absence d'excès de risque. En juillet 2002, deux plaintes pour homicide involontaire et pour non-respect de la réglementation relative aux installations classées sont déposées par l'association «Environnement 56 » qui se constitue partie civile, pour le compte de l'ambulancier, lequel décède en 2004. En 2003, un débat a lieu dans le Sud-est du Morbihan sur l'implantation d'un incinérateur de traitement des ordures ménagères. Nivillac y devient le symbole local des risques de santé associés à cette technique. Le cas de Nivillac se caractérise par une présence importante dans les médias en 2002, et par une très faible mobilisation des acteurs locaux. L'ouverture d'une procédure judiciaire en fait un cas spécifique par rapport aux deux autres ${ }^{11}$.

\section{Les conditions de formation du signalement}

L'étude des trois cas permet de caractériser le moment où le signalement se constitue, c'est-à-dire le moment où deux phénomènes déjà identifiés, une source de pollution et des problèmes de santé, sont mis en relation. C'est à partir de là qu'il est possible d'identifier les conditions dans lesquelles une épidémiologie populaire peut se déployer.

La condition première de formation d'un signalement est l'existence d'atteintes de santé perçues comme atypiques. Le caractère atypique recouvre deux aspects: la population touchée et l'absence d'explication plausible. Il mobilise des représentations culturelles des causes des maladies. Incontestablement, le fait que ce soit des cancers d'enfants constitue un facteur déterminant pour que se forme une représentation de pathologies inhabituelles auxquelles une explication courante ne peut pas être apportée. Dans les entretiens réalisés, le facteur déclenchant est une succession de cas de cancers divers dans un réseau lâche d'interconnaissance. Le biais rétrospectif des entretiens n'occulte pas le processus de

\footnotetext{
${ }^{11}$ Des dissensions au sein de l'association ont conduit son avocat à se retirer du dossier. Mais formellement la plainte est toujours instruite.
} 
construction du signalement qui résulte d'une absence d'explication causale de pathologies qui sont individuellement perçues comme inhabituelles et qui, ensemble, deviennent un agrégat atypique. Le contraste entre Nivillac et les deux autres sites illustre le facteur déterminant que représente le caractère atypique des pathologies. Dans cette zone à la démographie vieillissante, l'idée de l'exceptionnalité des cancers d'adultes ou de personnes âgées est en effet peu plausible quand le cancer, pris comme un tout, est une pathologie courante et une cause majeure de décès. Une des difficultés consiste à faire valoir localement l'exceptionnalité de ces décès. Les acteurs locaux ont alors recours à une procédure d'amplification du problème par une agrégation de tous les cancers qu'ils peuvent recenser. Une autre stratégie aurait pu consister à mettre en avant des situations exceptionnelles. L'attention des médecins qui ont relayé le signalement avait en effet été attirée par l'existence de cancers atypiques chez les deux membres de quelques couples, ce qui était inhabituel dans leur pratique professionnelle. Le signalement n'a pas mobilisé ces cas spécifiques, mais il a mis en avant l'ampleur des cancers ordinaire pour en construire l'aspect inhabituel.

Pour qu'un signalement prenne forme, il faut également que la connaissance de ces atteintes de santé puisse être rassemblée et cumulée afin de les construire comme un problème collectif. La structure des réseaux sociaux locaux est un vecteur déterminant dans cette collectivisation des pathologies. Contrairement aux deux autres sites, l'enquête menée à Saint-Cyr-l'École n'a pas permis d'identifier un moment fondateur du signalement. Deux enfants décèdent, l'un en 1996, l'autre en 1998 ; l'année suivante, deux autres enfants sont atteints de pathologies rares. L'information commence à circuler à la sortie des écoles, à l'initiative de trois mères, qui se sont déjà inquiétées des effets des antennes de radiotéléphonie sur le toit du groupe scolaire. Une préoccupation relative à ces antennes prend corps par l'intermédiaire des associations locales de parents d'élèves. Elle rejoint des revendications environnementales déjà anciennes concernant l'aménagement du quartier et l'extension de l'autoroute qui l'enserre. C'est par ces différents niveaux que la préoccupation acquiert une expression publique. Le rôle des associations de parents d'élèves est également déterminant à Vincennes pour amplifier un premier signalement par la directrice de l'école maternelle. Ces associations sollicitent des explications auprès de la Mairie. Elles bénéficient pour cela de l'appui d'un toxicologue, très engagé dans l'association de défense des victimes de l'amiante (l'Andeva), dont le siège se trouve à deux pas de l'école. Le problème posé est très largement rendu public par la sollicitation de différents relais associatifs ou institutionnels. A Nivillac, le signalement provient d'un ambulancier dont l'entreprise se trouve en situation de quasi monopole du transport sanitaire dans cette zone. Transportant les malades vers les centres de traitement des cancers, sa situation professionnelle lui permet de cumuler les cas de pathologies locales. Mais il n'est pas en capacité de mobiliser des réseaux locaux pour donner un retentissement à l'agrégat qu'il perçoit. L'autre signaleur, qui prend le relais de l'ambulancier, est récemment installée dans la région ; les réseaux locaux auxquels il est étranger lui sont fermés lorsque son action remet en cause les élus qui ont géré l'incinérateur. Dans les deux premiers clusters, le signalement passe par une mobilisation de réseaux sociaux faiblement organisés, réceptifs à des préoccupations concernant les enfants ; à Nivillac au contraire, l'absence d'accès à des réseaux locaux consolidés conduit les initiateurs du signalement à se reporter vers des médecins qui, en conflit avec les élus locaux, vont relayer le signalement auprès de l'autorité sanitaire.

Une troisième dimension réside dans la capacité d'imputer une cause plausible à ces pathologies. Il faut donc que le milieu environnant offre des ressources pour cette imputation. Les trois signalements mettent en cause des installations à caractère industriel qui présentent alors une relative invisibilité. Dans deux cas, ces installations ont cessé de fonctionner. A Nivillac, l'action en justice a interdit aux élus locaux de détruire l'incinérateur, comme ils 
entendaient le faire ; mais sa visibilité est réduite du fait de sa localisation dans des landes boisées à l'écart des habitations. Son impact sur l'environnement est de surcroît nul en comparaison des années où il enfumait le voisinage. A Vincennes, l'usine Kodak a été remplacée par un ensemble résidentiel; le passé industriel est seulement perceptible aux résidents par les noms des rues et quelques objets urbains qui renvoient au passé du lieu. A Saint-Cyr-l'École, les antennes sont banalement inscrites dans le paysage. Ainsi, lors des entretiens, plusieurs personnes ont affirmé ne pas les avoir remarquées avant le signalement. C'est en interrogeant leur cadre de vie et y cherchant des réponses à des questions non résolues que les personnes sont conduites à mettre en évidence ces installations comme des sources possibles de pollution à même d'expliquer les agrégats de pathologies atypiques. La mise en cause des installations est facilitée par l'absence d'interdépendance entre les plaignants et les responsables de la source incriminée ${ }^{12}$. Les résidents de Saint-Cyr-l'École et de Vincennes n'entretiennent pas de relations de dépendance ou de réciprocité avec les entreprises qu'ils mettent en cause. A l'inverse, à Nivillac, la mise en cause de l'incinérateur incrimine immédiatement des élus locaux qui maîtrisent les réseaux de sociabilité et de pouvoir local. De ce fait, la question posée par le signalement est vite transformée en enjeu politique local dans lequel les fidélités sont mises à l'épreuve. Dans les deux autres cas, le signalement prend une toute autre forme dans laquelle la mise en cause de la source de pollution n'interfère pas avec les conflits politiques locaux. Les maires, nouvellement élus, agissent en médiateurs entre les plaignants et les autorités de santé.

\section{La démarche de l'épidémiologie populaire}

La relation de causalité entre l'agrégat de pathologies et la source incriminée procède d'une intuition qui devient plausible par l'intermédiaire de différentes phases communes aux trois signalements étudiés. Ces phases permettent de caractériser la démarche engagée dans l'épidémiologie populaire des clusters.

L'intuition de l'existence d'un cluster repose très largement sur des hypothèses de contiguïté spatiale que l'utilisation de cartes de localisation des cancers permet d'objectiver. Dans le cas de Nivillac, il s'agit d'un assemblage de cartes d'état-major au $1 / 10000^{\mathrm{e}}$ de la région sur laquelle figurent des points rouges figurant les personnes décédées d'un cancer et des points bleus figurant des personnes malades. La carte comporte plus de 200 points répartis en nuages au nord de Nivillac et surtout à l'est. Au centre figure l'incinérateur. Elle a été constituée par l'ambulancier et complétée par les médecins locaux; elle a servi de document de travail pour l'enquête menée par la CIRE en 2002. Elle agrège les cas de cancers sur plus de dix années, indépendamment des types de cancer, de leur date d'apparition ou de diagnostic, et des caractéristiques des personnes. Elle établit une représentation spatiale qui objective le cumul des cancers. Elle est aussi la trame d'un récit, maintenant celui de la veuve de l'ambulancier chez qui elle est gardée, qui explique le déplacement progressif des cas de cancers de l'ouest vers l'est autour de l'incinérateur. Constamment réactualisée, elle est le registre spatial des cancers locaux. La carte a également été utilisée comme outil d'objectivation à Saint-Cyr-l'École pour localiser les cancers et pour délimiter les lieux situés à une certaine distance des antennes afin d'y réaliser des enquêtes. A Vincennes, l'exiguïté des lieux et le caractère diffus de la pollution chimique n'ont pas rendu nécessaire l'établissement d'une carte.

\footnotetext{
${ }^{12}$ La place de ces relations d'interdépendance dans la mise en cause a été soulignée dans différentes études relatives aux pollutions, en particulier Zonabend (1989), Wynne (1996), Van Staevel (2006).
} 
La représentation spatiale est un support intermédiaire dans l'établissement d'une relation de causalité : elle délimite un agrégat spatial et permet d'objectiver l'intuition initiale sur la cause des pathologies. Un travail documentaire permet de donner corps à cette première relation de contiguïté spatiale. Les connaissances mobilisées concernent les sources possibles de pollution par des produits chimiques ou des radiations. Elles s'alimentent à de la documentation accessible dans le monde scientifique ou dans la mobilisation environnementale (Medline, sites dédiés aux questions de santé environnementale, aux radiations électromagnétiques ou à l'incinération). A Vincennes, une source majeure des connaissances développées par les acteurs locaux provient d'un scientifique investi de façon militante dans le champ de la santé publique. La valeur attribuée aux connaissances qu'il diffuse dépend de la confiance qui s'est localement instaurée avec lui. Parmi les éléments sur lesquels repose cette confiance, il y a le jugement porté sur son expérience personnelle et la conviction de son désintéressement, c'est-à-dire la conviction de l'absence d'instrumentalisation du signalement dans des enjeux extérieurs à la situation locale. Une autre source de connaissance est constituée par le comité scientifique établi pour étudier le signal sanitaire, auquel participe un temps le toxicologue. Ce comité fournit des connaissances et des données qui acculturent le collectif local au raisonnement et aux résultats des études menées et lui permet également de développer une critique méthodologique (Ledrans et al., 2007). A Saint-Cyr-l'École, l'expertise institutionnelle ne s'articule pas avec le signalement local même si des instances de concertation sont prévues. Les enquêtes locales sur les troubles associés aux sources électromagnétiques sont disqualifiées par les experts. Les acteurs locaux font appel à des spécialistes critiques des radiations électromagnétiques, engagés dans les campagnes contre les antennes. Mais dès que Saint-Cyr-l'École est érigé comme cas exemplaire, des tensions se font jour dans la mobilisation locale et neutralisent cette montée en généralité. A Nivillac, outre un travail de recherche documentaire sur l'incinération, l'élu local à l'origine du signalement fait réaliser sur ses ressources propres, des mesures directes des polluants contenus dans le sol aux alentours de l'incinérateur. Durant l'expertise commanditée par le Ministère de la santé et réalisée sous le contrôle du préfet du Morbihan, il n'y a pas d'échanges qui puissent être une source d'informations pour les acteurs locaux. Dans chaque site, les démarches sont spécifiques: les sources documentaires qui alimentent le passage de l'intuition à l'établissement d'une causalité dépendent étroitement des configurations sociales dans lesquelles tant le signalement que l'expertise s'opèrent et des ressources qu'elles procurent aux acteurs locaux.

Des figures de rhétorique permettent de rassembler les diverses informations collectées et de les mettre en relation avec l'expérience locale des pathologies pour valider la causalité envisagée. Le raisonnement local fait ainsi appel à des synecdoques : à Saint-Cyr-l'École, le danger perçu des téléphones portables sert d'argument pour souligner les dangers des antennes. D'autres procèdent de principes d'identité : à Nivillac, l'incinérateur de Gilly-surIsère et son fonctionnement hors normes servent de matrice pour repenser les dangers de l'incinérateur. D'autres figures procèdent de la pensée analogique. Ainsi, dans le cas de SaintCyr-l'École, on invoque un exemple largement diffusé par les opposants aux ondes électromagnétiques, selon lequel les ondes ont des effets délétères chez les poussins et peuvent produire des malformations; la conclusion qui en est tirée est que ces ondes ou des ondes comparables devraient aussi avoir des effets identiques sur les enfants. A Nivillac, les tumeurs cancéreuses du chien de l'ambulancier sont invoquées pour attester la dangerosité de l'incinérateur et de ses émissions. Les rapports de comparaison ou de similitude que ces figures engagent sont utilisés à l'intérieur des réseaux qui signalent les clusters; ils se développent d'autant plus que l'expertise institutionnelle est faiblement présente pour les critiquer. Dès lors que ces figures rhétoriques sont considérées comme allant de soi, elles rendent plausible la causalité invoquée. Il convient alors de s'interroger sur les processus par 
lesquels elles vont de soi. Comparer un poussin et un enfant peut en effet avoir du sens au sein d'une famille dotée d'un langage et de catégories qui lui sont propres. Au-delà de ce cercle intime, cette comparaison mobilise des principes d'identité (œuf - fœetus ; poussin enfant) qui ne vont pas de soi dans les classifications admises. Comme le souligne Mary Douglas (1999), ces principes doivent être soutenus par un groupe social qui les tient pour admis, ce qui le conduit à les inscrire dans un ordre naturel des choses au-delà de toute contestation.

Ainsi, la démarche de l'épidémiologie populaire que l'on voit en œuvre dans ces trois sites part d'une intuition initiale qui s'appuie sur des faits immédiats avérés qui sont construits en tant que problème au sein d'un collectif de pensée. Elle met en œuvre une collecte locale de données qui vise à connaître l'étendue factuelle du problème considéré. L'intuition est alors étayée par des arguments théoriques collectés selon les ressources auxquelles les signaleurs ont accès. Cette démarche est descriptive, inductive et non contradictoire. Elle est à l'opposé de la démarche analytique des experts qui s'appuie sur des données issues de cohortes pour statuer sur les cas locaux considérés. Elle rejette les approches probabilistes et les explications multifactorielles des pathologies parce qu'elles remettent en cause la logique d'accusation qui est au fondement du collectif de pensée. C'est ainsi que l'organisation sociale des collectifs qui portent le signalement est déterminante tant dans la démarche mise en œuvre que dans le caractère plausible accordé à la relation de casualité construite.

\section{L’épidémiologie populaire comme expertise}

L'épidémiologie populaire s'alimente à la critique de l'expertise institutionnelle et de ses choix méthodologiques qui sont à l'opposé de ceux qu'elle met en oeuvre. La définition des objets d'étude est l'objet de critiques dès lors qu'elle ne correspond pas aux causalités mises en avant dans le signalement. La délimitation spatiale et temporelle des agrégats de cancers donne également lieu à contestation. Les profanes s'acculturent ainsi à l'épidémiologie savante et à l'expertise institutionnelle. Mais ce n'est pas à partir de cet apprentissage qu'ils revendiquent une prise de parole et une participation aux débats sur le cluster. S'ils remettent en cause les choix opérés, ils le font au nom d'une connaissance et d'une expérience de terrain. Cette expérience fonde leur revendication à l'expertise qui revêt des modalités différentes.

Dans ses recherches sur les mouvements sociaux relatifs à la santé environnementale, Sylvia Noble Tesh distingue deux stratégies d'expertise que les profanes peuvent adopter. Dans une première stratégie qu'elle qualifie de politique désintéressée, "les citoyens présentent leurs revendications comme si elles étaient libres d'intérêts égoïstes ou d'engagements personnels » (Tesh, $2000: 100)$. Les enjeux de pouvoir, de justice ou d'équité posés par les problèmes de santé environnementale sont déplacés vers des questions de recherche scientifique. La science est le moyen d'éviter les biais dans les décisions publiques. Cette stratégie conduit à confier l'élucidation des questions posées par les problèmes de santé environnementale à une communauté scientifique réputée libre de tout intérêt partisan et qui devient l'arbitre pour juger de la validité des revendications locales. Dans ce cas, les experts qui ne corroborent pas les intuitions ou les hypothèses sur lesquelles se fondent leurs revendications locales, peuvent être taxés de pratiquer une science impure prise dans un tissu d'intérêts politiques et d'enjeux de pouvoir. Une stratégie alternative consiste " à ancrer les revendications dans une connaissance issue de l'expérience des gens ordinaires qui vivent dans des endroits pollués. Ils parlent de sens commun, d'intuition, de ce qui est "évident" pour ceux qui vivent leur vie. Leur stratégie est une variation de la politique de l'identité 
commune aux mouvements pour l'égalité sociale » (Ibid. : 102). L'idée centrale est que les personnes qui sont exposées à la pollution sont dans une situation singulière, différente du reste de la population et que leur expérience leur donne une compréhension du problème posé. Elles revendiquent alors une connaissance engagée qui leur procure une expertise singulière, concrète, spécifique à laquelle n'accède pas la connaissance scientifique. Elles cherchent à dépasser la relation entre l'exposition à des nuisances et les problèmes de santé, qu'elles considèrent acquise, pour faire valoir l'apport de leur expérience.

Les trois clusters étudiés présentent des profils différents au regard de ces deux stratégies. Le cas de Nivillac se rattache sans aucun doute le plus à la stratégie désintéressée. L'élu local qui a porté le signalement au long des années passées revendique d'agir au nom du bien public et des intérêts des «pauvres gens » malades ou décédés du cancer qui, selon lui, n'ont ni les ressources, ni les moyens de se plaindre. Très engagé affectivement dans ce combat contre les politiques qu'il accuse de négligence et de concussion, il fait appel à une expertise scientifique validée par les réseaux écologiques. Par ailleurs, il soutient le recours en justice qui, selon lui, est apte à dire la vérité du cluster. Il se trouve ainsi contraint par cette stratégie lorsque les expertises ne sont pas concluantes ou que les procédures judicaires ne débouchent pas. L'adoption de cette stratégie est à la mesure de son isolement local ; comme il ne bénéficie pas de relais, ni de soutiens locaux, il n'est pas en mesure de faire valoir d'autres arguments relatifs au danger des incinérateurs pour la santé.

La mobilisation à Saint-Cyr-l'École se situe sur le registre de l'identité. C'est le collectif qui, fort de son expérience, revendique contre les antennes et leurs effets sur la santé. Cette expérience n'est pas directement celle des cancers; les familles endeuillées participent en effet peu à la mobilisation locale. Elle trouve son ancrage dans une identification à l'expérience de mères d'enfants malades par l'intermédiaire des associations de parents d'élèves. La distribution aléatoire des cancers sert de ferment à la constitution d'une réalité collective, l'exposition à des risques de santé liés aux antennes de radiotéléphonie,. La relation avec l'expertise institutionnelle est inévitablement conflictuelle puisqu'elle remet en cause la réalité du risque autour duquel se forme la mobilisation et qu'elle ne permet pas à leur expérience commune de mères d'enfants exposés aux risques d'être entendue.

A Vincennes, les experts scientifiques sont présents dès le début du signalement et mettent en œuvre une démarche active d'investigation. Le modèle qui prévaut alors est celui du désintéressement dans lequel la charge de la preuve revient aux experts. La pertinence de ce modèle suppose une confiance dans les experts, d'où les stratégies de communication que ces derniers déploient. Mais cette stratégie se trouve soumise à une double contrainte de répondre aux attentes locales relatives à la réalité du risque et de faire comprendre les doutes et les incertitudes qui traversent l'expertise. La mise en cause profane du désintéressement passe par la critique de la neutralité des experts dont certains, qui dépendent de Kodak, sont présentés comme juges et partie et donc intéressés. C'est à partir de là que le modèle de l'expérience est mobilisé pour critiquer l'expertise scientifique. En faisant appel aux connaissances qu'il a de la situation de terrain et en mobilisant des raisonnements courants sur la contamination et les délais de latence, le collectif considère que les estimations de risques sont minimisées (Lapides, 2007). L'appel aux savoirs locaux lui permet de critiquer l'expertise institutionnelle. Mais l'hétérogénéité sociale du collectif et les ambiguïtés relatives aux émanations incriminées dans les cancers ne lui permettent pas de faire valoir une expérience partagée de risques de santé comme source d'une expertise profane.

Dans ces trois situations, les acteurs locaux revendiquent une expertise qui devrait être prise en compte dans la décision publique. Le savoir qu'ils mobilisent est très différent de celui qui est construit par l'expertise officielle. Il prend très peu appui sur des données 
collectées dans des protocoles contrôlés, ne serait-ce que parce que les acteurs locaux n'ont ni le temps, ni les moyens d'utiliser ces protocoles et qu'ils mobilisent les ressources auxquelles ils ont accès. Il s'ancre dans une expérience locale et dans la connaissance qu'elle génère. Il s'oppose à la connaissance issue de l'expertise officielle. Cette opposition n'obéit pas à un modèle unique, mais elle dépend des possibilités pour faire valoir une revendication relative au problème posé par des cancers atypiques. A Nivillac, l'appel à une expertise scientifique extérieure répond à l'impossibilité de rassembler des acteurs locaux autour d'une cause qui ne peut pas s'appuyer clairement sur une expérience collective du risque ou de la maladie et qui met en cause des réseaux sociaux établis. A Saint-Cyr-l'École, le recours à l'expérience procède d'une mise à distance de l'expertise officielle et la prise en compte de connaissances issues d'expertises alternatives. Le cas de Vincennes offre une configuration intermédiaire dans laquelle les plaignants sont associés à l'expertise. Les conflits de perspective se dédoublent entre des conflits d'experts qui se concluent par la démission du toxicologue, et des conflits entre résidents. Ces derniers opposent le collectif Vigilance Franklin à une association de voisinage, composée de personnes anciennement implantées et qui voient d'un mauvais œil la publicité donnée à leur quartier. La Mairie se trouve au carrefour des différentes perspectives divergentes et en produit une articulation au travers des décisions qu'elle prend et des actes qu'elle engage.

\section{Conclusion}

Revenant sur les études qu'il avait menées à Woburn, Brown explique qu'il avait alors introduit la notion d'épidémiologie populaire pour qualifier «de façon spécifique les efforts de terrain engagés par les citoyens et impliquant des activités comme la 'cartographie profane', lorsque les résidents élaborent des cartes locales de maladies et vont de porte à porte pour recruter des voisins. Les profanes recrutent souvent des scientifiques pour les aider à déterminer des causes possibles[...] L'alliance sciences-citoyens est une approche plus institutionnalisée, et ne requiert pas que les profanes participent à toutes les phases du travail. Ainsi, l'épidémiologie populaire est un sous-ensemble des alliances sciencescitoyens »(Brown, 2007 : 34). En prenant appui sur trois signalements de clusters, c'est la dimension cognitive de cette épidémiologie populaire et la mobilisation locale qui la soustend qui a été privilégiée. Cette approche, centrée sur l'épidémiologie populaire en tant que mode de connaissance et d'explication des maladies, laisse donc en arrière-plan une analyse des relations entre ces signaleurs et des scientifiques mandatés ou s'engageant dans les signalements.

Le choix de travailler sur des signalements de clusters environnementaux oriente également le type de connaissances qu'il est possible de produire. Contrairement à d'autres clusters, par exemple de pathologies infectieuses, le chercheur est confronté à des situations dans lesquelles l'établissement d'une relation causale est difficile, sinon impossible à établir. Cette situation extrême est intéressante à analyser car tout oppose l'expérience locale et l'expertise scientifique: le type de données mobilisées, le mode de raisonnement, l'établissement de causalités, l'administration de la preuve. Les antagonismes entre un raisonnement local, qui procède du «bricolage » au sens l'entend C. Lévi-Strauss (1962: 26 et sq), et un raisonnement professionnel ne renvoient pas simplement à l'opposition entre le profane et l'expert, mais elles se rapportent également aux styles de travail qui caractérisent l'épidémiologie descriptive et l'épidémiologie analytique. Les terrains d'études conduisent ainsi à traiter une épidémiologie populaire particulière, relative au rôle de l'environnement 
industriel dans la genèse de pathologies non infectieuses, et non pas l'ensemble des pratiques de l'épidémiologie populaire.

Dans ces limites, on peut remarquer que l'épidémiologie populaire est diverse, à la mesure de la diversité des situations et des configurations sociales et politiques qui se forment localement. Elle prend appui sur ces cas avérés de maladie ou de mort pour produire une explication causale permettant d'ancrer des demandes de protection à l'égard de risques insidieux. Elle s'alimente aux incertitudes et aux limites de l'expertise épidémiologique auxquelles elle oppose la valeur de l'expérience de terrain. Malgré la proximité avec le langage et les catégories de l'épidémiologie scientifique, cette épidémiologie populaire en est très différente parce que les connaissances tacites qui sous-tendent la connaissance ne sont pas les mêmes ${ }^{13}$. En témoigne par exemple la notion d'aléa qui, pour les uns, est une explication, éventuellement par défaut, qui obéit à un ensemble de règles de la statistique, et qui pour les autres est une notion incompréhensible parce qu'elle ne fournit pas une explication certaine.

Poser aussi radicalement les différences entre les deux types d'épidémiologie conduit à s'interroger sur la pertinence de forums hybrides (Callon et al., 2001) dans lesquels les différents protagonistes seraient conduits à expliciter les fondements de leurs représentations des problèmes posés et, le cas échéant, à parvenir à une solution. Le cas de Vincennes est érigé en modèle de référence de ces forums par l'expertise officielle. Cette dernière peut trouver dans le type de concertation mis en œuvre un moyen de refonder une confiance dans sa capacité d'expertise en explicitant ses méthodes de travail et ses démarches (Brücker, 2007). Les deux autres cas renvoient à des situations qui sont plus complexes dans la mesure où les acteurs locaux ne s'inscrivent pas dans le cadre de référence épidémiologique proposé par les experts. Ils partent d'autres prémisses et le signalement d'un cluster leur permet d'exprimer publiquement des préoccupations relatives à la santé et aux risques qui ne seraient pas audibles autrement. C'est cette dimension, délaissée par les experts préoccupés de faire valoir la légitimité et la validité de leur démarche, qu'il convient d'interroger pour aborder la possibilité d'une résolution pragmatique des tensions et conflits dont les signalements de clusters sont l'expression.

Plusieurs décès d'enfants ou une succession de pathologies ont un impact émotionnel dans un ensemble social caractérisé par une certaine interconnaissance. Mais cela ne suffit pas à expliquer pourquoi des gens se mobilisent, consacrent des ressources en temps, en relations, jusqu'à parfois subvertir leur vie ordinaire. Cela n'explique pas non plus pourquoi d'autres les rejoignent et agissent avec eux. Pour aborder ces questions, le choix des trois sites s'avère $a$ posteriori intéressant. Dans deux sites, une mobilisation collective se développe; dans le troisième, elle échoue. Indépendamment de la structure des réseaux sociaux il convient de remarquer qu'à Nivillac, une critique sociale dont le cluster serait l'expression peut difficilement prendre appui sur la présence de pathologies courantes chez les personnes âgées parce que ces pathologies sont interprétées comme relevant de la nature des choses. Dans les deux autres sites au contraire, une critique sociale peut prendre appui sur l'argument de l'existence de pathologies chez des enfants, parce que ces pathologies n'entrent pas dans un ordre normal des choses dans le contexte français actuel. C'est cette situation qui nous semble la plus intéressante à examiner pour discuter de ce qu'expriment ces signalements de clusters.

Le trait saillant des deux signalements de cancers d'enfants réside dans la place centrale occupée par le couple «mère-enfants » dans la mobilisation, alors que les pères sont très largement absents ${ }^{14}$. C'est au nom de leurs enfants que les femmes se mobilisent ; c'est aussi

\footnotetext{
${ }^{13}$ Sur les connaissances tacites dans la confrontation entre expérience et expertise, Collins et Evans (2007).

${ }^{14}$ Le couple « mère-enfant » se retrouve également dans le reportage télévisé consacré à l'incinérateur de Gillysur-Isère et diffusé sur Antenne2 le 30 janvier 2002. Ce récit filmique est structuré autour du lait (des mères
} 
en leur nom qu'elles donnent une forme spécifique à cette mobilisation, refusant une montée en généralité ou une exemplarité de leur situation. Une analyse classique conduirait à dire que ces mères assument un rôle traditionnel de protection de leur progéniture. Il nous semble que cette explication ne convient pas pour des femmes qui ont un capital social et culturel élevé et qui, après avoir élévé leurs enfants, cherchent à retrouver une activité professionnelle.

Une explication alternative consiste à aborder cette mobilisation des mères par la question des risques et des réponses qu'il leur est possible d'apporter. Par un processus d'identification dont on a souligné le substrat social, elles se trouvent confrontées directement à la question de l'exposition de leurs enfants à des risques de santé dont elles ont une perception confuse. Les experts disent que le risque n'est pas supérieur à ce qui est normalement attendu ; mais leur connaissance locale des pathologies les conduit à remettre en cause cette affirmation et à ne pas faire confiance aux experts. Elles se trouvent ainsi dans une situation dans laquelle les institutions collectives remettent en cause ou invalident leur perception de dangers qui touchent l'intimité de leur vie familiale et des liens avec leurs enfants. Elles doivent alors se référer à elles-mêmes pour décider de ce qui est bon pour leurs enfants, de ce qu'il conviendrait de faire devant ces risques, puisque les institutions ont incapables de leur fournir des réponses ${ }^{15}$. Le risque de santé qu'elles perçoivent, en concernant les enfants, touche au lien de filiation autour duquel la vie familiale s'est organisée $^{16}$. Il se développe dans le contexte d'un tournant biographique marqué par la scolarisation des enfants et la recherche d'un nouvel emploi pour leurs mères. L'une des réponses apportée à ces configurations émotionnelles est de produire une action collective organisée à partir d'une condition similaire d'exposition aux risques. Cette action collective leur permet de partager leurs incertitudes, de s'extraire partiellement de la réflexivité biographique générée par l'entrée des risques dans leur intimité familiale. Elle est également le moyen par lequel des revendications de protection de l'intimité peuvent être formulées et entendues dans l'espace public. Tant que l'expertise officielle ne prend pas la mesure de cet ancrage des perceptions des risques de santé environnementale dans les sphères de l'intimité, elle ne peut pas entendre ce que dit cette épidémiologie populaire environnementale.

\section{Références bibliographiques}

Borraz O., Devigne M., Salomon D., 2004, Controverses et mobilisations autour des antennes relais de téléphonie mobile, Paris, Centre de sociologie des organisations.

Brown P., 1992, Popular epidemiology and toxic waste contamination: lay and professional ways of knowing, Journal of Health and Social Behaviour, vol. 3, 267-281.

Brown P., 1995, Popular epidemiology, toxic waste and social movements, In : Gabe J. ed, 1995, Medecine, health and risk. Sociological approaches, Oxford \& Cambridge, Blackwell, Sociology of health and illness monograph series, 91-112.

Brown P., 2007, Toxic exposures. Contested ilnesses and the environmental health movement, New York \& Chichester, Columbia University Press.

Brown P., Mikkelsen E.J., 1990, No safe place: Toxic waste, leukemia and community action, Berkeley, Los Angeles \& London, University of California Press.

\footnotetext{
allaitantes; on passe aux vaches et aux brebis productrices de lait et de fromage). Cette attention portée aux mères est d'autant plus remarquable que l'incinérateur est mis en cause pour des cancers touchant des adultes.

${ }^{15}$ Sur ces configurations relatives aux risques et à l'expertise, voir Giddens (1994).

${ }^{16}$ L'inscription du risque dans le lien de filiation différencie ces deux cas de Nivillac dans lequel les liens de l'alliance et les réseaux de parenté ont empêche le problème posé par les signaleurs d'acquérir une ampleur locale.
} 
Brücker G., 2007, Vers des démarches d'expertise partagée. Numéro thématique : cancers pédiatriques à Vincennes : quelles leçons tirer ? Editorial, $B E H, \mathrm{n}^{\circ}$ 7-8, 53-54.

Callon M., Lascoumes P., Barthe Y, 2001, Agir dans un monde incertain. Essai sur la démocratie technique, Paris, Le seuil, La couleur des idées.

Chateauraynaud F., Torny D., 1999, Les sombres précurseurs. Une sociologie pragmatique de l'alerte et du risque, Paris, Editions de l'EHESS.

Cicolella A., Benoit Browaeys D, 2005, Alertes santé. Experts et citoyens face aux intérêts privés, Paris, Fayard.

Collins H., Evans R., 2002, The third wave of sciences studies: studies of expertise and experience, Social studies of science, 32, 2, 235-296.

Collins H., Evans R., 2007, Rethinking expertise, Chicago \& London, University of Chicago Press.

Coura J.R., 2004, Epidémiologie, In : Lecourt D., dir, Dictionnaire de la pensée médicale, Paris, PUF, Quadrige, 425-429.

Dejour-Salamanca D, Gomes Do Espirito Santo M.E., Chappert J.L., Garcia S., Creteur X., Isnard H., 2005, Investigation d'un signalement d'agrégat de cancers de l'enfant à Saint-Cyrl'École, octobre 2004, BEH, n²9-50, 252-253.

Douglas M., 1999, Comment pensent les institutions, Paris, La découverte-MAUSS (Edition originale 1986).

Drass Ile-de-France, Cire Ile-de-France, Ddass Yvelines, InVS, 2004, Investigation du signalement d'un agrégat de pathologies diverses à Saint-Cyr-l'Ecole Département des Yvelines (78), Saint-Maurice, InVS.

Evans R., 2008, The sociology of expertise: The distribution of social fluency, Sociology Compass, vol. 2, 1, 281-298

Fleck L., 2005, Genèse et développement d'un fait scientifique, Paris, Les Belles Lettres, Coll. Médecine et sciences humaines (Edition originale 1935).

Giddens A., 1994, Les conséquences de la modernité, Paris, L’Harmattan, Théorie sociale contemporaine.

Hughes E.C., 1952, The sociological study of work, The American journal of sociology, vol 57,5, 423-426.

Hughes E.C., 1996, Le regard sociologique (Textes rassemblés et présentés par J.M. Chapoulie), Paris, Editions de l'EHESS.

Institut de Veille Sanitaire, 2002, Analyse d'un agrégat de cas de cancers dans l'école Franklin Roosevelt de Vincennes, Saint Maurice, InVS.

Institut de Veille Sanitaire, 2005, Guide méthodologique pour l'évaluation et le prise en charge des agrégats spatio-temporels de maladies non infectieuses, Saint Maurice, InVS.

Kroll-Smith S., Floyd H., 1997, Bodies in protest. Environmental illness and the struggle over medical knowledge, New York \& London, New York University Press.

Kuhn T., 1983, La structure des révolutions scientifiques, Paris, Flammarion (Edition originale : 1962).

Lapides V., 2007, Perception du dossier et de son déroulement par le Collectif Vigilance Franklin, $B E H, \mathrm{n}^{\circ} 7-8,59-60$.

Ledrans M., Hazebrouck B., Clavel J., Empereur-Bissonnet P., Cochet C., Fourme E., Garnier R., Goldschmidt F., Hartmann O., Jougla E., Lacour B., Lafon D., Momas I., Masse R, Ramel M., Suzan F, Zmirou-Navier D., Quénel P.., 2007, Regroupement de cancers pédiatriques à Vincennes, rôle du Comité scientifique de 2001 à 2006 : une confrontation entre les attentes sociales et l'expertise scientifique, $B E H, \mathrm{n}^{\circ} 7-8 ; 57-59$.

Lévi-Strauss C., 1962, La pensée sauvage, Paris, Plon

Parsons T., 1951, The social system, Free Press, New York. 
Robinson D., 2002, Cancer clusters : findings vs feelings, Medscape general medecine 4 (4). URL : http://www.medscape.com/viewarticle/442554 (dernier accès le 5 décembre 2008).

Salomon D, 2003, A l'opposé du principe de précaution. L'incinérateur de Gilly-sur-Isère, InVS, CIRE Rhône Alpes.

Tesh S. N., 2000, Uncertain hazards. Environmental activists and scientific proof, Ithaca \& London, Cornell University Press.

Thabuis A., Schmitt M., 2006, Usine d'incinération d'ordures ménagères de Gilly-sur-Isère (Savoie) Etude rétrospective d'incidence des cancers, CIRE Rhône-Alpes, DRASS RhôneAlpes, Institut de veille sanitaire.

Tillaut H., 2005, Recensement des agrégats de pathologies non-infectieuses, France, 19972002, BEH, 49-50.

Van Staevel E., 2006, La pollution sauvage, Paris, PUF Le Monde, Coll. Le partage du savoir.

Wynne B., 1996, May the sheep safely graze? A reflexive view of the expert-lay knowledge divide, In: Lash S., Szerszynski B., Wynne B. ed., Risk, environment and modernity. Towards a new ecology, London, Sage Publications, 44-83.

Zonabend F., 1989, La presqu'île au nucléaire, Paris, Editions Odile Jacob. 


\section{ABSTRACT \\ Lay reports of cancers clusters "Popular epidemiology" and expertise in environmental health}

Lay reports of cancers clusters combine the perception of an abnormal frequency of cancerous pathologies by a population, the incrimination of industrial facilities in these pathologies, and the report to health authorities for scientific investigations and control of health risks. Generally, the existence of a cluster is not validated by epidemiological investigation. The population is therefore led to develop its own knowledge of the health problem through a process that, following Phil Brown, we qualify as popular epidemiology. The article utilizes the ethnographic study of three reports of clusters in France (Saint-Cyr-L'École, Vincennes, Nivillac) to analyze this popular epidemiology, its claims to expertise, and its social and cultural foundations. 